\title{
Food choices of edentulous adults with implant- supported overdentures and conventional dentures
}

\author{
Do implant overdentures improve food choice and ability to chew food \\ compared with conventional dentures?
}

\section{Ellis JS, Thomason JM, Jepson NJ, NohI F, Smith DG, Allen PF. A randomized-controlled trial of food choices made by edentulous adults. Clin Oral Implants Res 2008; 19:356-361}

Design A randomised controlled trial (RCT) was carried out involving dental hospital patients.

Intervention The implant group (IG) were provided with conventional maxillary dentures and implant-retained mandibular overdentures and the denture group (DG) were provided with conventional dentures. Patients consumed seven test foods.

Outcome measure Pre- and post-treatment questionnaires were completed relating to food choices and chewing difficulty.

Results IG subjects reported increased consumption of carrots, apples and nuts post-treatment $(P<0.05)$ and decreased post-treatment difficulty in chewing apples and nuts. DG subjects reported decreased post-treatment difficulty in chewing carrots, bacon and nuts $(P<0.05)$. Between-group differences for chewing difficulty were detected for nuts, which DG subjects found easier to chew than did IG subjects $(P=0.002)$. Conclusions Food selection and perceived chewing difficulty improved in both groups, with no significant differences between groups. Successful rehabilitation may not result in different food selection, which may require concurrent tailored dietary interventions, but may increase available food choices.
Address for correspondence: JS Ellis, School of Dental Sciences, Newcastle University, Framlington Place, Newcastle upon Tyne, UK. E-mail: j.s.ellis@ncl.ac.uk

\section{Commentary}

This is the second RCT by these authors, dealing with more or less the same subject as their first paper ${ }^{1}$ but with a somewhat different purpose. This was clearly and eloquently stated: to assess the impact of implant-retained overdentures on the food selection of edentulous adults.

The inclusion and exclusion criteria of the participants were reasonably inclusive, although it would have been better to base the selection on the level of bone resorption rather than the duration of edentulousness. The randomisation process was well-defined and properly conducted. A power calculation was carried out to find the number of patients needed to detect differences between the groups. The study used the appropriate statistical tests for significance of changes to evaluate the before and after effects of the intervention.

Although the lack of masking (blinding) of both patients and providers might appear to be a negative property of the RCT, it is virtually impossible, in studies of this type, to mask the patient or the provider from the type of denture provided. A real negative, however, is that it is not clear what exactly the patients gave consent for: according to the authors, neither group was "aware of the other arm of the study": the participants did not consent to having implants and were not fully informed of the nature of the study.

This is also demonstrated by the fact that some patients in the IG refused the implant option after being randomly allocated to the group. To these patients, the intention-to-treat analysis (ITT) was applied. This was a good choice by the authors since ITT is typically applied to a group whose participants deviated from the treatment or were lost to followup. ITT should have also been applied, though, to the "five patients from the IG and the nine from the denture group" who were lost to followup at 3 months.

As described earlier, ${ }^{2}$ the design used in this study (where the participants are randomised before acquiring full consent; the Zelen design $)^{3}$ risks a high proportion of refusal which may affect the power of the study. Fortunately, this did not happen here, and the dropout rate was about $14 \%$.

Regarding the providers (prosthodontists), all we knew of them is that they were experienced. Nothing was mentioned about number of years of experience or calibration or standardisation of their work, although calibration in a study like this might be somewhat difficult.

It has been shown that implant-retained denture users are generally more satisfied than conventional denture users. ${ }^{4-6}$ With food selection and ability to consume hard or tough food being one aspect of patient satisfaction, this study showed that there was no difference in that aspect whether patients were using conventional complete dentures or implant-retained ones. This was explained through patient predisposition to using either type of dentures; ie, patients who are ready or willing to use conventional dentures will generally have improvement in their food selection and ability to consume hard food. The same is true for implant-retained denture seekers. 
The experience of the provider was another factor the authors used to explain their findings. The authors suggested that conventional DG participants may have had a different satisfaction level with the dentures should the dentures have been constructed by less experienced providers. Earlier, ${ }^{7}$ it was found that patients are generally satisfied with their implant-retained dentures regardless of level of experience of the provider.

The study will certainly help practitioners facing patients who refuse the implant-retained denture to demonstrate that quality of life can improve and satisfaction be achieved, as long as the conventional denture is properly constructed.

\section{Asim Al-Ansari}

Dental Department, Armed Forces Hospital, Dhahran, Saudi Arabia
1. Allen PF, Thomason JM, Jepson NIA, Nohl F, Smith DG, Ellis J. A randomized controlled trial of implant-retained mandibular overdentures. J Dent Res 2006; 85:547-551.

2. Jokstad A. Implant retained or conventional dentures, which give more patients satisfaction? Evid based Dent 2006; 7:96-97.

3. Zelen M. A new design for randomized clinical trials. N Engl J Med 1979; 300: 1242-1245.

4. Heydecke G, Thomason IM, Lund JP, Feine IS. The impact of conventional and implant supported prostheses on social and sexual activities in edentulous adults. Results from a randomized trial 2 months after treatment. J Dent 2005; 33:649-657.

5. Heydecke G, Penrod JR, Takanashi Y, Lund JP, Feine JS, Thomason JM. Costeffectiveness of mandibular two-implant overdentures and conventional dentures in the edentulous elderly. J Dent Res 2005; 84:794-799.

6. Awad MA, Lund JP, Shapiro SH, et al. Oral health status and treatment satisfaction with mandibular implant overdentures and conventional dentures: a randomized clinical trial in a senior population. Int J Prosthodont 2003; 16:390-396.

7. Esfandiari S, Lund JP, Thomason JM, et al. Can general dentists produce successful implant overdentures with minimal training? J Dent 2006; 34:796-801.

Evidence-Based Dentistry (2008) 9, 107-108. doi:10.1038/sj.ebd.6400611

\section{Key to evidence graphic used in the Evidence-based Dentistry Journal}

The graphic is based on the Centre for Evidence-based Medicine levels of Evidence tables www.cebm.net/levels_of_evidence.asp (see Evidence-based Dentistry 2003;4: p 17-18)

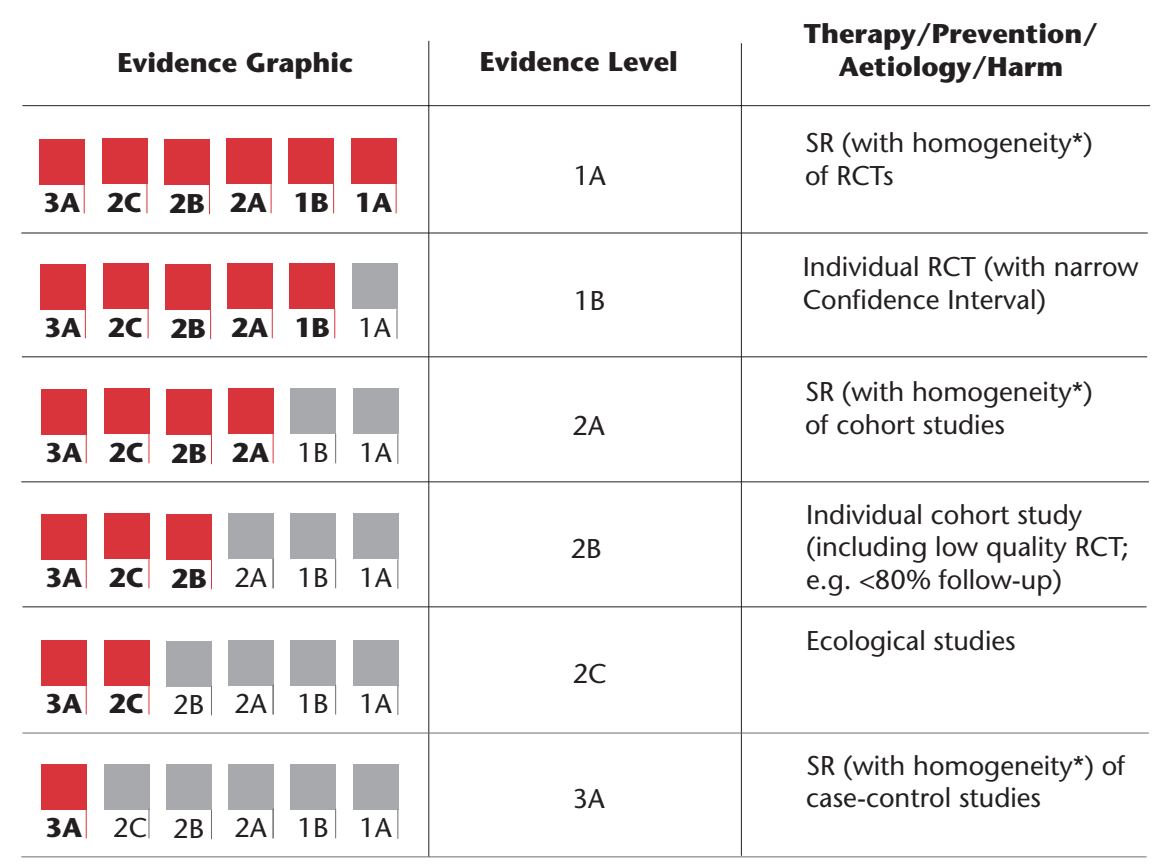

* By homogeneity we mean a systematic review that is free of worrisome variations (heterogeneity) in the directions and degrees of results between individual studies. Not all systematic reviews with statistically significant heterogeneity need be worrisome, and not all worrisome heterogeneity need be statistically significant. 